\title{
Index
}

Note: Page numbers with ' $\mathrm{t}$ ' are tables.

1951 Convention Relating to the Status of Refugees (and the 1967 Protocol) $37,124,125$

1954 Convention Relating to the Status of Stateless Persons 37

1961 Convention on the Reduction of Statelessness 37

1961 Vienna Convention on Diplomatic

Relations 43-44, 46

1967 Protocol 37, 125, 156

1969 American Convention on Human Rights 94, 128

2005 World Summit 68, 81, 91, 101, 108

academics 167,169

actors $7-9,13,14,15,173$

civil society 179

ad hoc international criminal tribunals 86 advocacy $134-136$

Africa 94-95

African Charter on Human and Peoples' Rights (Banjul Charter) 94, 128

African Commission on Human and Peoples' Rights 128

African Court on Human and Peoples'

Rights 94, 129

African Union (AU) 12, 83, 94, 128, 177

regional courts 129

Albright, Madeleine 48, 79

allies 158

American Convention of Human Rights (1969) 94,128

American Declaration on the Rights and Duties of Man (1948) 94

the Americas 93-94, 127-128, 129

amicus curiae 142-143, 161

Amnesty International 2, 138-139, 142

Clapper v. Amnesty International USA 142 letter to UN 143-145

Amos, Valerie 126

anarchy 20

Annan, Kofi 104

Global Compact initiative 9, 102, 179

In Larger Freedom 102

More Secure World: Our Shared

Responsibility, A 101-102

Arab Charter on Human Rights 95, 128

Arab Human Rights Committee 95, 128

Arab League 95, 177

arbitration 14

Arbour, Louise 104-105

artists 169

ASEAN see Association of South East Asian Nations

Asia, IGOs 95

Assad, Bashar al- 36, 85

Assange, Julian 61

assimilation 124

assistance, humanitarian 15

Association of South East Asian Nations (ASEAN) 12, 95, 129, 177

Intergovernmental Commission on Human Rights 128

AU see African Union

authoritarianism 56,175 ,

$$
\text { 176-177, } 178
$$

Axworthy, Lloyd 47-48

Ayala-Lasso, Jose 103

Bahrain 44

Balkans 68, 76-77, 79, 159

Banjul Charter 94, 128

Ban Ki-moon 102, 126, 143

Bashir, Omar el- 86

Beneson, Peter 138

Bessler, Manuel 158

bilateral diplomacy 47 
Boutros-Ghali, Boutros 103

Agenda for Peace, An 101

Burke, Jason 45

Bush, George W. 91, 140

business 178-179

\section{Cairo Declaration on Human Rights in} Islam 95

Canada 47-48, 62

capitalism 25-26

Carter, Jimmy 31-34

cause lawyers 161

celebrity diplomacy 137

charter-based bodies 113

China 173, 174, 176

Balkans 79

Internet 176

as Most Favored Nation 30-31

Resolution 68876

citizen diplomacy 137-138

civil service

OCHA 125-126

regional 126-129

UN High Commissioner for Human

Rights 102-113, 121

UN Secretaries-General 100-102

civil society actors $1,9,11,56,67,134$, 163,173

civil society space 55,56

Clapper v. Amnesty International USA 142

Clausewitz, Carl von 21

Clinton, Bill 30-31

Clinton, Hillary 61

Cluster Coordination approach 126

Coalition for the International Criminal Court 136

colonialism see imperialism

commission diplomacy 12, 80

Commission on Human Rights 89-91

commissions, national human rights $61-62,63$

commissions of inquiry 121

committee diplomacy 12-13, 123

Common Article 3 (Geneva Conventions) 37

conference diplomacy 11-12

Constantine, Greg 167-169

constructivism 27 controversy of human rights 4-6

Convention on the Prevention and the Punishment of Genocide 37

Convention on the Prohibition of the Use, Stockpiling, Production and Transfer of Anti-Personnel Mines and on their Destruction (Ottawa Treaty) 48, 137

Corporate Social Responsibility (CSR) 9, 102

Costello, Joe 53-58

Council of Europe 93, 127

counter-summits 11

"Country Reports on Human Rights

Practices" (US State Dept) 34

courts/tribunals $86,94,174$

ICC 15, 83, 94-95, 136

regional 128-129

CSR see Corporate Social Responsibility

cultural relativism 5

Daalder, Ivo 48

Dayton Peace Accords 77

démarches 58, 60

democracy 26, 177

Democratic People's Republic of Korea

(DPRK) 53-54

d'Escoto Brockman, Miguel 88

detention monitors 162-167

Diana, Princess of Wales 138

dignity see human dignity

diplomacy 2.0 10, 53

diplomacy

channels 10-13

heads of state 27-36

tools and strategies $13-15$

types $9-10,14,82-83,121,123,137-138$

see also international diplomatic law

diplomatic immunity 44

diplomatic recognition 44

Dunant, Henry 146

ECOSOC (Economic and Social Council) 89,91

Egeland, Jan 126

Emergency Relief Coordinator (ERC) 126

Ethiopia 150

European Convention on Human Rights and Fundamental Freedoms (1950) 93 
European Court on Human

Rights 93, 128

European Court of Justice 128

European Union (EU) 93

Annual Report on Human Rights and

Democracy 52

Balkans 80

regional civil service $126-127$

failed states $177-178$

field diplomacy 13, 155

negotiation 157-158

first/second/third generation human rights 3,6

first/second/third generation peacekeeping 68, 69-74t, 75, 173

first/second/third UN 67, 78, 81, 102

Follow the Money 148-149

foreign ministers/secretaries 47-48

foreign policy 1, 19, 21, 22, 23

United States 30-34

see also Ministries of Foreign Affairs (MFA)

Fox, Hosanna 147-150

Freedom House 176-177

freedom of movement 157

Frelick, Bill 139-142

Gaddafi, Muammar 83-84

Gayton, Ivan 164-165

Gebauer, Thomas 137

gender equality $57,107,162$

generations (first/second/third) of human rights 3,6

Geneva Conventions 7, 37, 39

ICRC 146, 162-163

genocidaires 78

Global Compact initiative 9, 102, 179

globalization 178-179

Global South 47, 87, 173

Goldstone, Richard 80

good offices 101

Goodwill Ambassadors 125

Gorbachev, Mikhail 29-30

Guterres, António 125

handbooks for human rights/humanitarian workers 156, 157-158 heads of state $20,27-36,31-32$

hegemonic power 21-22, 23

Helsinki Summit/Accords 28-29, 39, 139,173

Helsinki Watch 29

Hicks, Peggy 135

HRC see Human Rights Council

human dignity 2-4, 7, 16, 175-176

cultural/religious practices 5, 6, 180

detention 162, 166

Helsinki Accords 29

IHL 7

Marxism 26

humanitarian diplomacy 13, 15, 135

humanitarianism (defined) 7

humanitarian space 136

human-rights based approach 101

Human Rights Council (HRC) (UN) 89-92, 106, 109

special procedures $114,115-118 \mathrm{t}$

human rights (defined) 2-4

human rights officers 158-159

human rights reports 52

Human Rights Watch 29, 44, 45-46, 135, 139-142

From a Flood to a Trickle 141

letter to UN 143-145

Silent Treatment, The 140

human security 12, 48, 75, 101, 137

human security network 137

hybrid courts 86,174

ICBL see International Campaign to Ban Landmines

ICC see International Criminal Court

ICCPR see International Covenant on Civil and Political Rights

ICISS see International Commission on Intervention and State Sovereignty

ICRC see International Committee of the Red Cross

ICTR see International Criminal Tribunal for Rwanda

ICTY see International Criminal Tribunal for the former Yugoslavia

IDPs see internally displaced persons 
IGO diplomacy (network diplomacy) 11, 92-95, 100, 125, 129

and $\mathrm{OHCHR} 113$

regional 127

see also United Nations (UN)

IGOs see intergovernmental organizations

IHL see international humanitarian law

ILC see International Law Commission imperialism (colonialism) 26, 178

Independent International Commission on Kosovo (Kosovo Commission) 80

Independent Permanent Human Rights Commission 95, 128

India

Khobragade incident 44-46, 58

Ministry of External Affairs 47

Resolution 68876

InterAmerican Commission on Human Rights (1959) 94, 127-128

InterAmerican Court of Human Rights 94,129

intergovernmental organizations (IGOs) 8, $9,15,20,92-95,173$

see also European Union; IGO diplomacy; United Nations

internally displaced persons (IDPs) 125

International Bill of Rights 2, 127

International Campaign to Ban Landmines (ICBL) 137

international civil service 100

International Commission on Intervention and State Sovereignty (ICISS) 12, 81,82

International Committee of the Red Cross (ICRC) 2, 37, 145-147, 151, 160

detention monitoring 162-3

International Convention on the Elimination of All Forms of Racial Discrimination 3

International Covenant on Civil and Political Rights (ICCPR) 2, 7, 89

International Covenant on Economic, Social and Cultural Rights (ICESCR) 2, 89

International Criminal Court (ICC) 15, 83, 94-95, 136, 174

International Criminal Tribunal for the former Yugoslavia (ICTY) 77, 80, 86
International Criminal Tribunal for Rwanda (ICTR) 79

international diplomatic law 43-47

international humanitarian law (IHL) 7 , 13, 22, 36-39, 177

detention monitoring 162-163

landmines 137

negotiations 158

International Law Commission

(ILC) 160

international legal personality 19

International Red Cross and Red Crescent Movement 146, 156

international relations theory 19,20

Internet 145, 174, 176

intervention/nonintervention 4, 23, 35, 47, 81,173

Iran, and the US 46

Iraq

and Kuwait 75-76

refugees $140-141$

United States 76, 82, 91

Ireland, MFA 53-58

Israel 89

jails/prisons 166

Jefferson, Thomas 24

journalists 169

Kenya 82-83

Kenyatta, Uhuru 83, 86

Keynesian liberalism 24, 25

Khan, Irene 145

Khobragade, Devyani 44-46, 58

Kosovo Commission see Independent

Commission on Kosovo

Kuwait, and Iraq 75-76

landmines 137-138

language 46

lawyers 159-162, 169

League of Arab States 94

Leahy, Patrick 137

Lesbian, Gay, Bisexual, Transgender and Queer/Intersex (LGBTQ/I) 11, 57, $113,135,161,162,163,173$

letter-writing campaigns 138

leverage 158 
liberalism 23-25, 29, 179, 180

see also states, illiberal

Libya 83-85, 165

lobbying 142

loud/quiet advocacy 158

low politics 22

McHugh, Gerard 158

McMillion, Margaret 58-61

Malinowski, Tom 44

Mancini-Griffoli, Deborah, Humanitarian Negotiation 157-158

market sovereignty 179

Marxian worldview 25-26, 29

Marx, Karl 26

material assistance 158

Médecins Sans Frontières (MSF) 2, 145, 147-151

detention monitoring 164-165

Humanitarian Negotiations Revealed

(McHugh and Bessler) 158

media 121

see also social media

mediation 14

megaphone diplomacy 121

MFN see Most Favored Nation

Middle East, IGOs 94-95

Milosevic, Slobodan 79, 80, 105

Mine Ban Treaty see Convention on the Prohibition of the Use, Stockpiling, Production and Transfer of AntiPersonnel Mines and on their Destruction (Ottawa Treaty)

Ministries of Foreign Affairs (MFA) 47, 48-61, 62-63

US Department of State 48-52, $52 \mathrm{t}$

Most Favored Nation (MFN) status, China 30-31

MSF see Médecins Sans Frontières

Muller, Bobby 137

multilateral diplomacy 8, 129, 173

see also IGO diplomacy; United Nations Myanmar 83

naming and shaming 145, 178

national human rights commissions 61-62

NATO (North Atlantic Treaty Organization)
Balkans 48, 77, 79, 80

Libya 84

negotiation 157-158

neoclassical liberals 24-25

network diplomacy see IGO diplomacy

NGO diplomacy 8, 134-143, 173

nongovernmental organizations (NGOs)

$8-9,15$

experts $135-136$

Freedom House 176-177

Helsinki Watch 29

humanitarian 145-151

humanitarian diplomacy 13, 15

human rights 138-142

Human Rights Watch 29

Rwanda 78

third UN 67

see also NGO diplomacy

nonintervention/intervention 4, 23, 35, 47, 81,173

nonrefoulement 124

OAS (Organization of American States) 12, 93-94, 128, 129

OCHA (Office for the Coordination of Humanitarian Affairs) 125-126

Humanitarian Negotiations with Armed Groups (McHugh and Bessler) 158

O'Flaherty, Michael 156

OHCHR (Office of the High Commissioner for Human Rights) 8, 101, 102-113, 167

ombudsman offices 61-62, 63

Operation Turquoise 78

Organization of Islamic Cooperation (OIC) 94, 129, 177

Organization for Security and Cooperation in Europe (OSCE) 80, 93

Ottawa Convention 15

Ottawa Treaty 48, 137

the P-5 67-68, 81

and Secretaries-General 100-101

parallel conferences 11

peacebuilding $68,75,173,178$

Peacebuilding Commission 68,

101-102

peace enforcement 68,75 
peacekeeping 68, 69-74t, 75, 77, 173, 178

Peace of Westphalia 7-8

persona non grata (PNG) 44

Picot, Andre, Humanitarian Negotiation 157-158

Pillay, Navanethem (Navi) 105-112

Pinheiro, Paulo Sergio 89-91, 119-121

pledging conferences 11

power 21-22

preventative diplomacy $82-83$

prisons/jails 166

private diplomacy 10

professional expertise 158

professionalization of human rights/

humanitarianism 155-157, $167-169,170$

detention monitors 162-167

human rights officers 158-159

lawyers 159-162

negotiation 157-158

Pronk, Jan 126

propaganda 10

protests 11

public diplomacy 10, 114

heads of state $31-32$

Putin 35-36, 85

Robinson 104

Putin, Vladimir 35-36, 85

quiet diplomacy 10, 105, 114

quiet/loud advocacy 158

R2P see Responsibility to Protect (R2P)

Ramcharan, Bertrand 104, 119

rapporteurs, special 114, 115-118t, 119, 121

Pinheiro 89-91, 119-120

Reagan, Ronald 29-30

realism 20-23, 26, 29

Red Cross/Crescent see International Red Cross and Red Crescent Movement refugees

1951 Convention Relating to the Status of Refugees (and the 1967 Protocol) $37,124,125$

Iraqi 140

UNHCR 123-125, 140-141

regional approaches to human rights 92-95

regional civil service 126-129 repatriation, voluntary 124

reprisals 56

resettlement 124

Resolution 45/111 166

Resolution 688 75-76

Resolution 1973 72t, 83-84, 85

Responsibility to Protect (R2P) 81-87, 88, $89,108,174,178$

responsibility while protecting (RWP) 84

Robinson, Mary 9, 35, 103-104

Roth, Kenneth 145

Russia 173, 174

Balkans 79-80

human rights reports $52-53$

Rwanda 68, 76, 78-79, 80, 105

United States on 58-61

Saudi Arabia, Wallstrom incident 48

Sauerbrey, Ellen R. 141

Schultz, George 29-30

secretariats 100

secretaries see foreign ministers/secretaries

securitization of aid 136

security, human $12,48,75,101,137$

social media $10,53,145,174$

Somalia $76,77-78,80$

South Africa 87

sovereignty $4-5,22,35,47,81,173$

and the Helsinki Summit/Accords 28

ICC 86

imperialism 26

market 179

regional human rights 94

special rapporteurs see rapporteurs, special

Sri Lanka 151

standing 142

stateless people $167-168$

Statement of Ethical Principles for Human

Rights Professionals 157

states $7-8,9,14,19-27,177$

authoritarian 56, 175, 176-177, 178

development of international law 36-39

failed $177-178$

heads of $20,27-36$

illiberal 175-177

international diplomatic law 43-47

and NGOs 136, 142

structuralism, see Marxian worldview 
subjects of international law 19

Sudan 86, 102

summit diplomacy 10-11, 27-28

Sweden, MFA 48

Syria $54,85-86$

Putin on $35-36,85$

terrorism 174,178

theory 20, 27

track $1 / 2$ diplomacy $1,14-15,134$

treaties, human rights $3,37,38 \mathrm{t}$

monitoring bodies 121-123, $122 \mathrm{t}$

treaty-based bodies 113

treaty monitoring 2, 12, 113, 121, 122t, 123, $126-128,160$

trustee systems 178

truth commissions 62

UDHR see Universal Declaration of Human Rights

Ukraine 54

Ulrich, George 156

UNHCR (United Nations High Commissioner for Refugees)

123-125, 140-141, 160

detention monitoring 163-164

Monitoring Immigration Detention 165

United Kingdom (UK)

and Assange 61

Foreign and Commonwealth Office 47

Iraq 76, 82

Libya 84

Rwanda 60

United Nations (UN) 4, 8

Charter 4-5, 66-67

General Assembly 87-89

High Commissioner for Human Rights 102-113, 121, 143-145

HRC 89-92

OCHA 125-126, 158

OHCHR 8, 101, 102-113

Secretaries-General 100-102, 173

Security Council 62-68, 69-74t, 75-82, 173

courts $86-87$

R2P 81-86

Special Committee on Apartheid 87

treaty monitoring bodies 121-123, 122t

UNHCR 123-125
United States

and Boutros-Ghali 101

Department of Defense 51

Department of State 47, 48-52, $52 \mathrm{t}, 141$

detention monitoring by ICRC 163

foreign policy 30-31

as hegemon 21, 23

Helsinki Accords 29-30

and Human Rights Watch 140-141

international diplomatic law 43

Bahrain incident 44

and Iran 46

Khobragade incident 44-46

Iraq 76, 82, 91, 140-141

liberal worldview 176

Libya 84

realist worldview 23

regional human rights 94

Rwanda 58-61

Somalia $77-78$

Syria 85

Universal Declaration of Human Rights (UDHR) 2, 2-4, 37, $39,89,106,175$

universalism 5

Universal Periodic Review (UPR) 92, 106, 109, 110

victims of war 7

Vieira de Mello, Sergio 104

Vienna Convention see 1961 Vienna

Convention on Diplomatic

Relations

voluntary repatriation 124

Wallstrom, Margot 48

war 22, 46

World Trade Organization (WTO) 31

worldviews 13, 26-27, 39, 45, 80, 173

and the Helsinki Summit/Accords 29

liberalism 23-25

Marxian 25-26

realism 20-23

Yugoslavia see Balkans

Zeid al-Hussein, Prince 112-113 